\title{
Improving Reliable Multicast Using Active Parity Encoding Services (APES)
}

\author{
Dan Rubenstein, Sneha Kasera, Don Towsley, and Jim Kurose \\ Computer Science Department \\ University of Massachusetts at Amherst \\ \{drubenst, kasera, towsley, kurose\}@cs.umass.edu
}

\begin{abstract}
We propose and evaluate novel reliable multicast protocols that combine active repair service (a.k.a. local recovery) and parity encoding (a.k.a. forward error correction or FEC) techniques. We show that, compared to other repair service protocols, our protocols require less buffer inside the network, maintain the low bandwidth requirements of previously proposed repair service / FEC combination protocols, and reduce the amount of FEC processing at repair servers, moving more of this processing to the end-hosts. We also examine repair service / FEC combination protocols in an environment where loss rates differ across domains within the network. We find that repair services are more effective than FEC at reducing bandwidth utilization in such environments. Furthermore, adding FEC to a repair services protocol not only reduces buffer requirements at repair servers, but also reduces bandwidth utilization in domains with high loss, or in domains with large populations of receivers.
\end{abstract}

\section{INTRODUCTION}

Many applications require the reliable delivery from a single sender to a large number of receivers. Providing such a delivery in a best-effort network where packet losses are frequent, such as the Internet, requires a reliable multicast proto$\mathrm{col}$, whose specific function is to compensate for these losses. Because network bandwidth is a limited resource, there is considerable interest in improving upon the bandwidth utilization of such protocols, especially in the event that they must support delivery to thousands of receivers [12].

Repair services and hybrid parity encoding / automatic repeat request (FEC/ARQ for short) are two approaches which reduce the bandwidth requirements of reliable multicast protocols. The repair services approach utilizes repair servers, which localize retransmissions to regions of the network where loss occurs [4], [6], [7]. Because each repair server might support numerous sessions in the network, and has limited buffer to store packets for retransmission, it is important to reduce the per-session buffer requirements at each repair server.

FEC/ARQ is an end-to-end approach which uses erasure coding techniques to produce special repair packets [1], [2], [10]. Typically, the set of repair packets that can repair all losses incurred by receivers is smaller than the set of packets that need retransmission [8]. However, end-hosts must perform additional encoding or decoding operations.

Further reduction in bandwidth requirements can be accomplished by designing protocols that utilize both approaches [5], [9]. We refer to such protocols as Active Parity Encoding Services protocols, or APES protocols for short. In this paper, we introduce two novel APES protocols, which, compared to previ-

\footnotetext{
This material was supported in part by the National Science Foundation under Grant No. NCR-9508274, and NCR-9527163, and by DARPA under Grant No. N66001-97-C-8513. Any opinions, findings, and conclusions or recommendations expressed in this material are those of the authors and do not necessarily reflect the views of the National Science Foundation.
}

ous APES protocols, maintain similar bandwidth requirements and reduce buffering and FEC processing within the network. The reduction in buffer is achieved by restricting buffering to a small set of encoded repairs instead of buffering original data packets. This set of FEC repair packets can be used to repair many combinations of losses incurred by receivers and because the set is smaller than the set of original data packets, it utilizes less buffer. FEC processing is also reduced at repair servers in the network by moving the majority of such processing to the end-hosts of the network (sender and receivers).

We perform an analytical study of these new APES protocols to determine the network bandwidth and repair server buffer requirements as a function of a repair server's likelihood of successfully servicing a repair request. These requirements are compared to those of previous approaches (both non-FEC repair server and previous APES). We find that generating all repairs at the sender and forwarding them to repair servers allows repair servers to service repair requests with the same likelihood as in previous approaches, but with a smaller buffer. However, this forwarding causes a considerable increase in bandwidth between the sender and the repair server. We find that if repairs are generated and buffered at the repair server, then repair servers can service repair requests with the same likelihood using roughly the same amount of of network bandwidth as in previous approaches. However, the difference in buffer requirements is a function of the rate at which the sender transmits data, with the new approach yielding a larger savings as the transmission rate increases.

Finally, we determine how various loss characteristics within the network impact the bandwidth savings achieved by using an APES protocol instead of a protocol that uses repair service approaches without FEC, or only an end-to-end FEC/ARQ approach. For this study, we base our network models on studies which indicate that receiver loss rates vary across different regions of the network [3], [14]. We find that in such models, repair services provide more significant savings in bandwidth than end-to-end FEC/ARQ. However, we find that APES protocols, in addition to reducing buffer requirements, use noticeably less bandwidth than non-FEC repair server protocols in high loss regions, or in regions where a repair server services a large numbers of receivers.

The remainder of the paper proceeds as follows. The network topology and APES protocols are presented in Section II. The protocols' performance is analyzed in Section III. Section IV examines the impact that the networking environment has on the benefit of adding FEC/ARQ or repair services. Finally, we suggest directions for future work and conclude in Section V. 


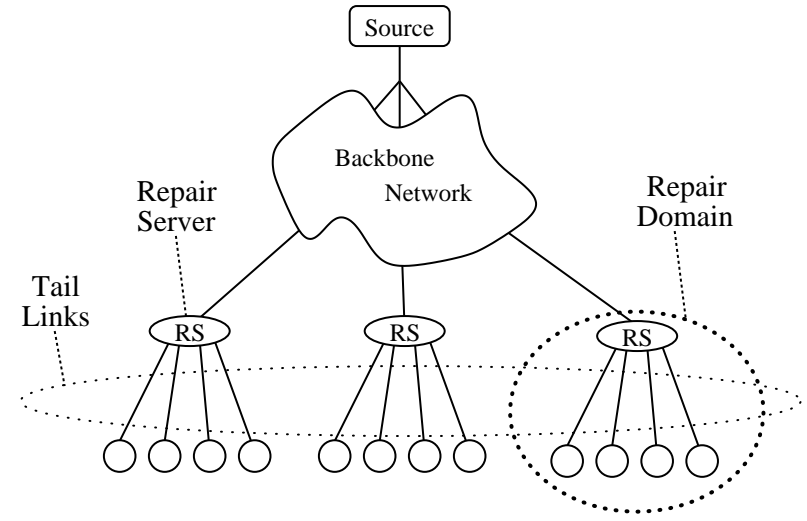

Fig. 1. The network model.

\section{APES}

Our network model consists of a multicast tree, which contains a sender at the root of the tree, and receivers at the leaves of the tree. ${ }^{1}$ Receivers are topologically partitioned into $N$ repair domains, $D_{1}, \cdots, D_{N}$. We define the domain size, $\left|D_{i}\right|$ to be the number of receivers in domain $i$. In this paper, we consider a one level hierarchy of repair servers, where each repair server is located at a point in the tree between the sender and a single domain. This allows the repair server to receive all packets that are multicast from the sender to receivers within its domain. The repair server can also subcast (multicast excluding the link toward the sender) packets to its repair domain. See Figure 1 for an example.

APES protocols send FEC-based repairs in place of retransmissions. The sender groups data packets into blocks of size $k$ (henceforth $k$ is referred to as the block size), and feeds them into an encoder to produce repair packets. The number of repair packets that can be produced is sufficiently large that, for our purposes, it is safe to assume that the sender can produce an unlimited supply. Repair packets generated from a block of $k$ data packets are said to belong to the block from which they were generated. Any entity (e.g. repair server or receiver) with a decoder can retrieve the $k$ data packets for a block once it receives any combination of $k$ data and repair packets that belong to that block. FEC-based protocols perform reliable delivery by ensuring that each receiver gets $k$ distinct packets per block. The fact that receivers can lose different data packets but use the same set of repairs to recover from losses reduces the number of required repair transmissions.

To ensure that each of its downstream receivers gets at least $k$ distinct packets per block, a repair server must receive $k$ distinct packets per block from the sender. We refer to the first $k$ packets received at the repair server per block (of block size $k$ ) as source packets, which can consist of any combination of data and repair packets belonging to that block. The various protocols use different combinations of buffering, forwarding, and FEC coding at the repair server to ensure reliable delivery of $k$ distinct packets to downstream receivers. We now consider what the repair server does in each protocol in order to ensure that a single block of packets is reliably transmitted to the receivers in its domain. Multiple blocks are delivered by applying the de-

\footnotetext{
${ }^{1}$ In practice, what we call a receiver is likely to be a local area network (LAN) that can contain several receiving applications.
}

TABLE I

HIGH LEVEL COMPARISON OF THE THREE PROPOSED APES PROTOCOLS.

\begin{tabular}{|l||c|c|c|}
\hline Repair Server... & SDBR & BRSR & GRSR \\
\hline \hline Encodes? & Yes & Yes & No \\
\hline Decodes? & Yes & No & No \\
\hline Buffers & Data & Repairs & Repairs \\
\hline Fwds repairs fr. src & Never & Sometimes & Always \\
\hline
\end{tabular}

scribed protocols to each block of data being transferred. Due to lack of space, specific feedback mechanisms are not presented here, but appear in [11].

The Store-Data-Build-Repairs Protocol (SDBR): This protocol is similar to previous proposals [5], [9]. Once a repair server reliably obtains $k$ source packets, it reproduces (via FEC decoding) the $k$ original data packets, which it subsequently buffers. Whenever an additional repair is required by one or more receivers, the repair server generates a distinct repair via FEC encoding.

The Build-Repairs-Store-Repairs Protocol (BRSR): A repair server decides in advance on a fixed number, $b$, of repairs per block to generate via FEC encoding. Here, the repair server does not buffer the source packets, but merely supplies them as they arrive to the FEC encoder. We refer to this coding process as on-the-fly encoding. ${ }^{2}$ For that block, only the $b$ packets that are generated are buffered at the repair server. These $b$ packets occupy buffer space upon arrival of the first source packet, and cannot be used for repair until the $k$ th source packet has been fed into the encoder. If all receivers lose less than $b$ source packets, then they can reconstruct the original data by reliably receiving a subset of repairs residing in the repair server's buffer. Reliability is guaranteed by having the repair server reliably transmit needed repairs, instead of generating new repairs for each loss. We shall see that this results in a reduction in buffer size and also in a negligible increase in bandwidth compared to the bandwidth used by SDBR. If any receiver loses more than $b$ source packets, it must obtain additional repairs from the source, since the repair server is unable to generate additional repairs.

The Get-Repairs-Store-Repairs Protocol (GRSR): Under this protocol, the repair server does not require FEC encoding capabilities. Instead, it requests $b$ repair packets from the sender, which it buffers. Once the repair server obtains the $b$ packets, the protocol behaves identically to BRSR for the remainder of the transmission of the block.

The value for $b$ can be chosen to be between 0 and the size of the block, $k,(0 \leq b \leq k)$. In Section III, we examine how the choice of $b$ impacts bandwidth and buffer requirements.

Table I presents a high-level comparison of the three proposed APES protocols. SDBR is the most efficient in terms of bandwidth, but its per block buffer requirements are identical to that of a non-FEC repair server protocol, henceforth referred to as an $R S$ protocol. It also performs decoding at repair servers. ${ }^{3}$ BRSR and GRSR buffer only repairs. Since receivers reliably

\footnotetext{
${ }^{2}$ Coding packages with on-the-fly encoding capabilities, as well as several other enhancements, and supporting documentation are available for download at http://www-net.cs.umass.edu//drubenst/software/software.html\#fec.

${ }^{3}$ The decoding is not a requirement of the protocol. However, previous work assumes the capability is available. If decoding is not performed, then SDBR will require receivers to perform the recursive decoding algorithm described in
} [11]. 
receive these repairs, there is never a need to buffer more than $k$ repairs per block. This means that these protocols will never buffer more packets per block than SDBR. They also do not perform decoding at repair servers. BRSR and GRSR differ in that BRSR requires additional FEC processing at repair servers, while the repair servers in GRSR perform no encoding. As a result, GRSR requires extra bandwidth to deliver repairs between the sender and repair server that BRSR instead generates with its encoder.

We assume, for BRSR and GRSR, that the repair server restricts buffering to the $b$ repairs. Other policies (such as adding additional repair transmissions from the source to the buffer when receivers require more than $b$ repairs), do not lead to considerable bandwidth savings, and can significantly increase the amount of buffer utilized. We will also assume only a single hierarchical level of repair servers. However, the protocols could easily be extended to include multiple hierarchical levels. These issues are addressed in [11].

\section{BUFFER/BANDWIDTH PERFORMANCE}

As a consequence of our model, each repair domain can be analyzed separately. Let us assume that a block size of $k$ is used, a repair server has $r$ receivers downstream, and each receiver loses any packet sent to it with a probability $p$. To simplify presentation, we do not concern ourselves with how the repair server reliably obtains $k$ source packets. Hence, we assume that there is no loss between the sender and the repair server. We consider four performance metrics: the expected number of packet transmissions from the repair server to its downstream receivers, the expected number of repairs received by the repair server from the sender, the expected number of packets that must be buffered per block at a repair server, and the repair server's expected buffer utilization at any moment in time, which we refer to as the buffer size.

We begin by analyzing BRSR and GRSR. We index the $k$ source packets that arrive at the repair server from 1 to $k$. Additional repairs are assigned distinct indices larger than $k$. We assume that the repair server multicasts all packet transmissions. Our analysis also assumes that a receiver that loses $m$ of $k$ packets in a block requires the repair server to reliably transmit packets $k+1$ through $k+m$. In practice, a receiver that requires $m$ repairs and loses some of the repairs numbered $k+1$ through $k+m$ could effectively use any repair numbered $k+m^{\prime}, m^{\prime}>m$ in place of a lost repair. By doing this, the receiver can only decrease the number of times it requests a particular repair. Thus, our assumption gives a conservative upper bound on the number of transmissions from a repair server.

To simplify presentation, we define $\gamma_{j}^{k}(p)$ to be the probability of losing exactly $j$ of $k$ packets.

$$
\gamma_{j}^{k}(p)=\left(\begin{array}{l}
k \\
j
\end{array}\right) p^{j}(1-p)^{k-j}
$$

Define $\phi_{i}$ to be the probability that a receiver requires transmission of packet $i$ within the block. For $i \leq k, \phi_{i}=1$. For $i>k$, this is the probability that fewer than $2 k-i+1$ of the initial $k$ packets are received by a receiver. If exactly $2 k-i+1$ of the initial $k$ packets are received, then it can recover the block by reliably obtaining the $i-k-1$ repairs numbered $k+1$ through $i-1$. Thus,

$$
\phi_{i}=\left\{\begin{array}{ll}
1, & i \leq k \\
1-\sum_{j=0}^{i-k-1} \gamma_{j}^{k}(p), & k<i \leq 2 k \\
0, & i>2 k
\end{array}\right\}
$$

Define $q_{i}(j)$ to be the probability that at least one receiver needs more than $j$ transmissions of packet $i$,

$$
q_{i}(j)=\left\{\begin{array}{ll}
1, & i \leq k, j=0 \\
0, & i \leq k, j>0 \text { or } i>2 k \\
1-\left(1-\phi_{i} p^{j}\right)^{r}, & k<i \leq 2 k, j \geq 0
\end{array}\right\}
$$

\section{A. Bandwidth: Repair Server to Receivers}

We now consider the expected bandwidth required between the repair server and its receivers (data plus repairs). Let $T_{S D B R}$, $T_{B R S R}$, and $T_{G R S R}$ be random variables that denote the number of packets that are transmitted by the repair server using SDBR, BRSR, and GRSR, respectively. The analysis presented in [8] that gives the bandwidth requirements between a sender and a set of receivers over a star topology lends itself directly to the bandwidth computation for SDBR in our network model:

$$
E\left[T_{S D B R}\right]=\sum_{j=0}^{\infty} 1-\left[1-\sum_{m=0}^{k-1} \gamma_{m}^{k+j}(1-p)\right]^{r}
$$

We now compute upper bounds on $E\left[T_{B R S R}\right]$ and $E\left[T_{G R S R}\right]$. Let $\tau_{i}$ be a random variable that equals the number of times that packet $i$ is transmitted. For $i \leq k$, we have $E\left[\tau_{i}\right]=1$, since the packet is always transmitted at most once. For $k<i \leq 2 k$, a packet is transmitted as many times as it is needed by some receiver. Hence, (recalling that our computations yield conservative upper bounds) we have

$$
\begin{gathered}
E\left[\tau_{i}\right] \leq \sum_{j=0}^{\infty} q_{i}(j) \\
E\left[T_{B R S R}\right]=E\left[T_{G R S R}\right] \leq k+\sum_{i=k+1}^{2 k} E\left[\tau_{i}\right] .
\end{gathered}
$$

Figure 2(a) presents the expected number of transmissions (normalized per packet) for a block size of 10 and receivers with loss rates of $5 \%(p=.05)$, as a function of the domain size. We observe a clear reduction in bandwidth due to the introduction of FEC, and further observe that BRSR and GRSR use essentially the same bandwidth as SDBR until the domain size grows very large. We emphasize that the bandwidth from the repair server to receivers is unaffected by the choice of $b$.

Figure 2(b) gives an upper bound to the ratio of the expected number of per packet transmissions for BRSR and GRSR over the expected number of per packet transmissions for SDBR as a function of block size. Here, there are 8 receivers in the repair domain. Curves are presented for various loss rates. We observe that, for loss rates at or below $10 \%$, SDBR is only $4 \%$ more bandwidth efficient than BRSR and GRSR. The difference is significant only for high loss rates. Thus, for reasonable loss rates, BRSR and GRSR do not use substantially more bandwidth than SDBR between the repair server and receivers. 


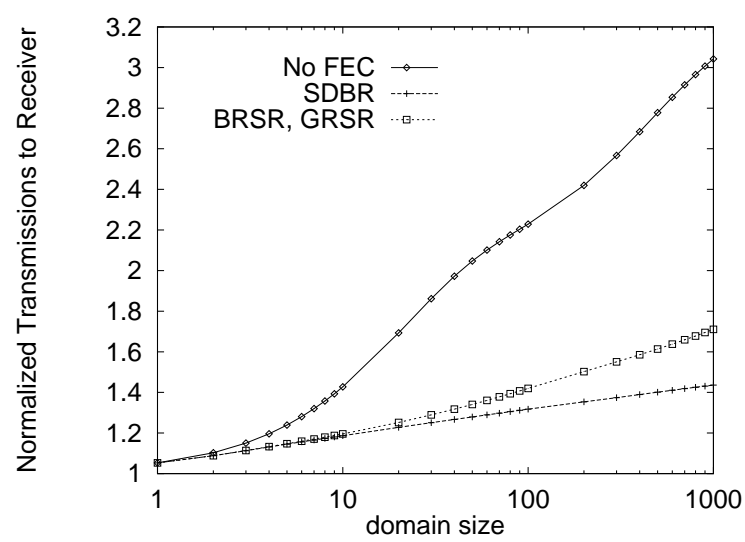

(a) Varying domain size

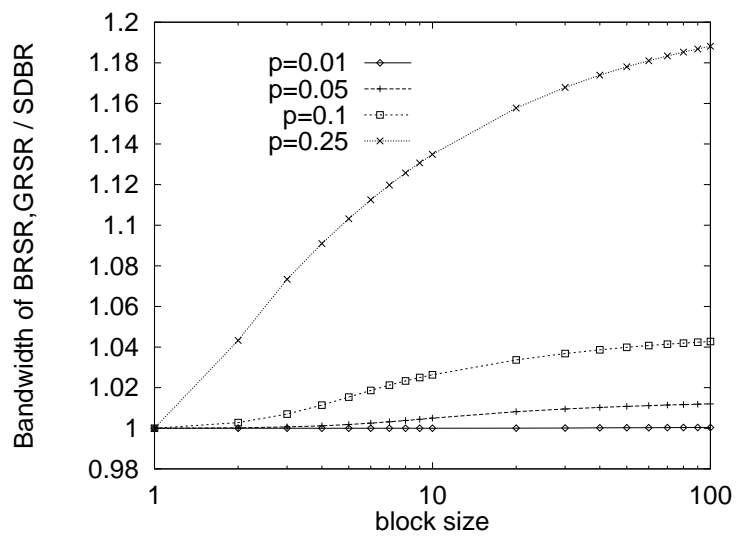

(b) Varying block size

Fig. 2. A bandwidth comparison of protocols.

Note that because SDBR always sends the minimal number of distinct repairs to provide reliability, it provides a lower bound on the expected bandwidth for BRSR and GRSR. The small difference in bandwidth required by SDBR when compared to the upper bounds of BRSR and GRSR indicates that the upper bound is tight. To summarize, SDBR requires less bandwidth between the repair server and receivers than BRSR and GRSR. However, for domain sizes and loss rates that one might expect in practice, the difference in bandwidth is negligible.

\section{B. Bandwidth: Sender to Repair Server}

Recall that for BRSR and GRSR, if receivers lose more than $b$ source packets, then additional packets must be obtained from the source by the repair server. Let $A$ be a random variable equal to the number of additional transmissions the sender must make to a repair server. The approach used to derive (2) yields the following:

$$
E[A]=\sum_{i=k+b+1}^{2 k} E\left[\tau_{i}\right] .
$$

The expected number of repairs that the sender must reliably send to a particular repair server under BRSR is bounded from above by $E[A]$. For GRSR, where the $b$ buffered repairs are transmitted from the sender as well, the expected number of repairs is bounded from above by $b+E[A]$.

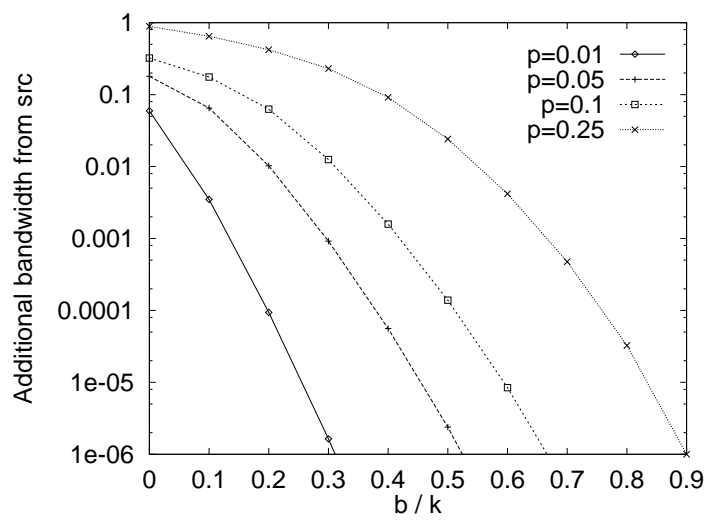

Fig. 3. Additional packets from insufficient buffering, normalized to block size.

We now focus on how the choice of $b$ affects per-block buffer and sender-to-repair server bandwidth requirements. The graph in Figure 3 illustrates the expected additional bandwidth normalized per packet (i.e., $E[A] / k$ ) as a function of the normalized initial buffer size (i.e., $b / k$ ) for various loss rates. The block size is 10 and the domain size is 8 . This illustrates that the number of times that the repair server must retrieve a repair from the sender is extremely small when $b$ is chosen sufficiently large. From this, we conclude that for sufficiently large $b$, the additional bandwidth needed from the sender is negligible. We see a similar trend as we vary the block size or the domain size (see [11]).

\section{Expected Buffer Size}

We refer to the size of the buffer being utilized by the protocol at any given time as the buffer size. The expected buffer size is an increasing function of both the number of packets that must be buffered, and the amount of time that each packet is buffered. We define $B_{R S}$ to be the buffer size of an RS protocol, and $B_{B R S R}$ to be the buffer size of BRSR. We now compute $E\left[B_{R S}\right]$ and $E\left[B_{B R S R}\right]$. Later, we discuss how these values compare with the buffer size of GRSR, $E\left[B_{G R S R}\right]$.

Our analysis of the BRSR and RS protocols considers a repair server to which data packets arrive with arrival rate, $\Lambda$. We also assume that there is a fixed round trip time (RTT), $R$, between each receiver and its upstream repair server, which includes the time that it takes for a receiver to detect a packet loss, send feedback to the sender, and have the sender respond with retransmissions. When a repair server cannot provide sufficient repairs for a block (because its buffer does not contain sufficient information to produce repairs for that block), we say that a repair server miss has occurred for that block. For the RS case, a repair server miss is defined per packet (i.e., a block size of 1). We measure the repair server's effectiveness in terms of the repair server miss probability, $\mathcal{P}_{k}$, which is defined to be the probability that at least one repair server miss occurs for a block of size $k$. Given $k$, we can compare expected buffer sizes by choosing some $\epsilon_{k}$ and determining the expected buffer size so that $\mathcal{P}_{k}<\epsilon_{k}$.

A difficulty in comparing buffer sizes for differing block sizes 
is that repair server misses are defined in terms of the block size. A similar difficulty arises when comparing buffer sizes for an APES approach with an RS approach. However, we can take advantage of the fact that we assume losses in the network are described by a Bernoulli process so that, for the RS protocol, $\mathcal{P}_{1}<\epsilon_{1} \Leftrightarrow \mathcal{P}_{k}<\epsilon_{k}$ whenever $\epsilon_{k}=1-\left(1-\epsilon_{1}\right)^{k}$. We perform a fair comparison over different block sizes, $k_{1}$ and $k_{2}$ by selecting a value, $\epsilon_{1}<1$. From this, we compute $\epsilon_{k_{1}}$ and $\epsilon_{k_{2}}$ using the above equivalence relation.

The buffer requirements that would allow a repair server to have a miss probability lower than an application chosen bound, $\epsilon_{1}$, were computed in [4] for an RS protocol. The repair server maintains each original data packet in its buffer for a fixed number of transmissions, $N$, which allows each receiver at least $N$ attempts at receiving the packet. The value of $N$ is chosen so that $\mathcal{P}_{1}=1-\left(1-p^{N}\right)^{r}<\epsilon_{1}$, where $p$ is the loss probability from the repair server to each receiver, and $r$ is the domain size. Using Little's Law, the expected buffer size can be computed:

$$
E\left[B_{R S}\right]=\Lambda R(N-1)
$$

The term, $\Lambda R$ indicates the number of packets that a sender is expected to send between receiver retransmission requests. We refer to this value as the retransmission factor.

For protocol BRSR, we compute an upper bound on the time that each packet is maintained in the buffer, such that the repair server miss probability, $\mathcal{P}_{k}$, is less than an application chosen bound, $\epsilon_{k}$, for a block size of $k$. A repair server miss occurs when either $b$ repairs are insufficient for some receiver, or when one of the $b$ repairs is released from the buffer before it it received by some receiver that required it. Define $N_{i}$ to be the number of times that a receiver can request transmission of packet $i$ (waiting one RTT between transmission requests) before the repair server drops packet $i$ from its buffer. We have $N_{i}=0$ for $i \leq k, i>k+b$. We now compute values of $N_{i}$ for $k<i \leq k+b$. Define $\psi(j)$ to be the probability that a receiver loses $j$ source packets and makes a request for a repair that is not in the buffer. This will occur if $j>b$, or if both $j \leq b$ and some packet $k+m$ is not received after $N_{k+m}$ transmissions, $1 \leq m \leq j$

$$
\psi(j)=\left\{\begin{array}{ll}
\gamma_{j}^{k}(p)\left[1-\prod_{m=1}^{j}\left(1-p^{N_{k+m}}\right)\right] & 1 \leq j \leq b \\
\gamma_{j}^{k}(p) & j>b
\end{array}\right\}
$$

Define $\psi$, to be the probability that a receiver requires a packet that causes the repair server to request an additional packet from the sender.

$$
\psi=\sum_{j=1}^{k} \psi(j)
$$

The repair server miss probability, $\mathcal{P}_{k}$, equals the probability that a repair server needs to request an additional repair from the sender. This equals the probability that at least one downstream receiver requires such a packet.

$$
\mathcal{P}_{k}=1-(1-\psi)^{r}
$$

Thus, it is sufficient for a repair server to choose $b$ and the set of $\left\{N_{i}\right\}$ sufficiently large so that $\mathcal{P}_{k}<\epsilon_{k}$.

We now determine the expected buffer size. Each repair packet $i, k<i \leq k+b$, begins to occupy buffer space once the first source packet for the block arrives at the repair server. To be conservative, we assume that no feedback is sent from receivers until the $k$ th source packet has been transmitted by the repair server, and has been given ample time (e.g., half a RTT) to be received. ${ }^{4}$ Afterwards, the $i$ th repair packet is held for a period of time that allows receivers $N_{i}$ attempts at retrieving it. The rate at which the $i$ th packet arrives (or is constructed) at the repair server is $\Lambda / k$. Buffer space is used by the $i$ th packet during the period of time it is being constructed (i.e., the time between the arrival of the first and $k$ th source packet), plus enough time to allow $N_{i}$ retransmissions to receivers. The expected amount of time that packet $i$ resides in the buffer is $(k-1) / \Lambda+R N_{i}$. It follows from Little's Law that the expected amount of buffer being used to hold packet $i$ over all blocks is $(k-1) / k+\Lambda R N_{i} / k$. The total expected buffer size is obtained by summing over the $b$ values of $i$ for which packets can reside in the buffer:

$$
E\left[B_{B R S R}\right]=\frac{b(k-1)}{k}+\frac{\Lambda R}{k} \sum_{i=k+1}^{k+b} N_{i} .
$$

Figure 4 demonstrates how the retransmission factor affects the expected buffer size for various values of $\epsilon_{1}$. The $x$-axis indicates the retransmission number, and the various curves represent the values of $\epsilon_{1}$. In this figure, the loss rate is .05 , and the domain size is 8 . Figure 4(a) gives the expected amount of buffer for an RS protocol. We see that for a small retransmission factor, very little buffer is required. The buffer size increases linearly with the retransmission factor.

Figure 4(b) gives the ratio of the expected buffer of BRSR with a block size of 10 to that of the RS protocol. We see that for a small retransmission factor, the expected buffer of the RS protocol is actually less than that of BRSR. This is due to the fact that as the data rate slows, the RS protocol can make more retransmissions of a given packet before BRSR (and similarly, SDBR) receives $k$ source packets and is able to complete its building of repairs. Thus, very low rate data transfers via SDBR or BRSR make inefficient use of the buffer. We see in Figure 4(b) that as the retransmission factor increases, BRSR's buffer increases at a slower rate, and buffer size is considerably smaller for high rate data flows.

Figure 5 gives the expected buffer size for BRSR as a function of the retransmission factor for a variety of block sizes. A small block size results in a smaller buffer size for a small retransmission factor, because large block sizes must store repairs that are in the process of being built for longer periods of time. Their increase in efficiency at repairing loss makes them more effective as the retransmission factor increases.

We have seen that the retransmission factor is a critical factor in determining how effective BRSR can be at reducing buffer requirements. Several additional factors lead us to believe that our computations produce an upper bound on the buffer size used by BRSR. For instance, if packet transmissions from the sender

${ }^{4}$ Note that receivers could potentially send feedback sooner. For example, a loss of the first source packet could be detected long before the $k$ th source packet arrives. 


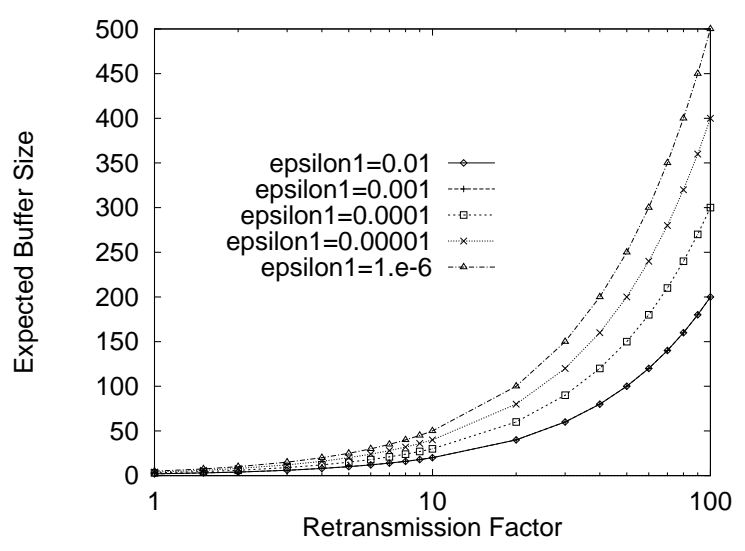

(a) Expected buffer size, using an RS protocol

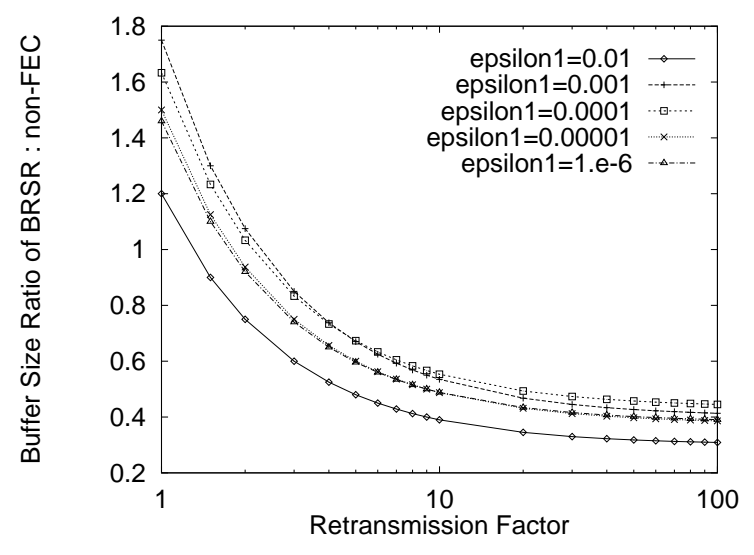

(b) Ratio of expected buffers of BRSR to RS.

Fig. 4. Expected Buffer Size comparison

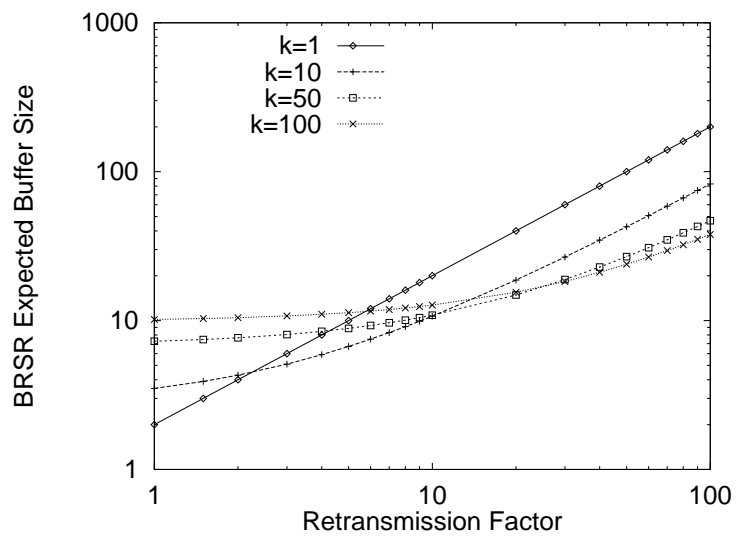

Fig. 5. Expected BRSR buffer for various block sizes.

are bursty, or if receivers transmit feedback in bursts (e.g., the bit-vector approach in the RMTP protocol [7], [13]), buffer size at the repair server for RS protocols will increase to retain the packets at the start of the burst. However, bursts will have no impact on buffer size in BRSR so long as each burst is subsumed within a block.

Finally, we discuss the buffer requirements of GRSR. We note that the buffer requirements differ in that repair packets are not cached in advance of receiving the $k$ th source packet. We conservatively assume that all repairs arrive immediately after the last source packet for the block. ${ }^{5}$ Little's law gives the expected buffer size to be:

$$
E\left[B_{G R S R}\right]=\frac{\Lambda R}{k} \sum_{i=k+1}^{k+b} N_{i} .
$$

The ratio of expected buffer size of GRSR to an RS protocol remains constant as the retransmission factor varies. Since the values of $N$ and $\left\{N_{i}\right\}$ are chosen independent of the retransmission factor, it turns out that

$$
E\left[B_{G R S R}\right] / E\left[B_{R S}\right]=\lim _{R \Lambda \rightarrow \infty} E\left[B_{B R S R}\right] / E\left[B_{R S}\right] .
$$

The ratio of expected buffer size of GRSR to an RS protocol can be viewed as the asymptotic values of the curves in Figure 4(b) as the retransmission factor increases.

In summary, we have analyzed the buffer and bandwidth requirements for various APES protocols. For domain sizes and loss rates that one would expect in practice, bandwidth requirements of BRSR are similar to those of SDBR, and BRSR also uses considerably less buffer than SDBR. The bandwidth requirements of GRSR on links between the repair server and receivers are identical to those of BRSR. However, because encoding is not performed at the repair server, additional bandwidth is used between the sender and repair servers to transmit all repairs which are to be buffered. GRSR uses a fraction of the buffer that an RS repair services protocol uses to meet a fixed miss probability. BRSR uses more buffer to meet a fixed miss probability for slow data rates. However, as the data rate increases, the buffer requirements of BRSR approach those of GRSR, and are considerably less than the buffer requirements of RS repair server protocols and of SDBR.

\section{APES-FAVORABLE ENVIRONMENTS}

We now consider the reduction in network bandwidth that results from using APES protocols. We also reveal a network setting which frequently occurs in real networks that we believe causes the largest variation in the amount of savings in bandwidth that is obtained by using an APES approach.

We extend our network model to consider receivers with different loss rates. Define $\mathcal{R}_{i, j}$ to be the $j$ th receiver in domain $i$, $1 \leq j \leq\left|D_{i}\right|, 1 \leq i \leq N$. We define $P_{i, j}$ to be the loss probability observed by receiver $\mathcal{R}_{i, j}$. Without loss of generality, we assume that receivers are enumerated within each domain, $i$, so that for any $1 \leq j<j^{\prime} \leq\left|D_{i}\right|, P_{i, j} \leq P_{i, j^{\prime}}$, i.e., receivers are ordered within each domain by increasing loss rate.

We define a multicast session to be intra-homogeneous if, for any $i$ and for all $j, j^{\prime}, P_{i, j}=P_{i, j^{\prime}}$, i.e., all receivers in the same domain have the same loss rate. A session that is not intrahomogeneous is intra-heterogeneous. We define a session to be inter-homogeneous if for any two domains, $D_{i}$ and $D_{i^{\prime}}$, we have that $\left|D_{i}\right|=\left|D_{i^{\prime}}\right|$ and for each $j \leq\left|D_{i}\right|, P_{i, j}=P_{i^{\prime}, j}$. If a session is not inter-homogeneous, it is defined to be interheterogeneous.

\footnotetext{
${ }^{5}$ If packets arrive out of order at the repair server (i.e., repairs arrive before source packets), the repair server can send the repairs as source packets and use the late source packets as repairs.
} 
We also assume that the sender always multicasts repairs, and the repair server always subcasts repairs. For simplicity, we again consider a network where all losses occur on the tail links, which lie between each receiver and its repair server (again, the reader is referred to Figure 1). This means that receiver losses are independent from one another, and that there are no losses within the backbone network (i.e., no losses between the sender and the repair server).

An examination of inter-homogeneous domains appears in [9]. Loss studies over the MBone [3], [14] suggest that loss rates differ in different regions of the Internet. Additionally, the domain size can vary considerably over the set of domains in a session. Consequently, we expect most multicast sessions to be inter-heterogeneous. We now examine an inter-heterogeneous, intra-homogeneous session, in which there are two types of domains. There are $n_{i}$ domains of type $i$, where each domain of type $i$ consists of $r_{i}$ receivers, each having a loss rate of $p_{i}$, $i=1,2$. In this domain, we consider two protocols.

e-e: The e-e protocol is a hybrid, end-to-end FEC/ARQ protocol that does not make use of repair servers. The protocol details and its analysis can be found in [8].

SDBR: SDBR is described in Section II. By setting the block size to one, this protocol performs identically to an RS repair server protocol.

Our measure of bandwidth is the number of packets (data plus repairs) that are sent per block of data. We define the random variable, $N_{i}^{S}$, to equal the number of packets multicast from a repair server to downstream receivers for a block in SDBR. We extend (1) so that it applies to the various types of domains $(i=1,2)$ :

$$
E\left[N_{i}^{S}\right]=\sum_{j=0}^{\infty} 1-\left[1-\sum_{m=0}^{k-1} \gamma_{m}^{k+j}\left(1-p_{i}\right)\right]^{r_{i}}
$$

We define $N^{E}$ to be a random variable that equals the number of (data and repair) transmissions in the e-e protocol that a sender must transmit to all downstream receivers (over all domains). Then

$$
E\left[N^{E}\right]=\sum_{j=0}^{\infty} 1-\prod_{i=1}^{2}\left[1-\sum_{m=0}^{k-1} \gamma_{m}^{k+j}\left(1-p_{i}\right)\right]^{r_{i} n_{i}}
$$

We also define $F_{i}$ to a random variable that equals the number of transmissions in a domain of type $i$ by a repair server using a protocol that does not implement FEC. $E\left[F_{i}\right]$ equals $E\left[N_{i}^{S}\right]$ when $k=1$, and can be written more succinctly as

$$
E\left[F_{i}\right]=\sum_{j=0}^{\infty} 1-\left(1-p_{i}{ }^{j}\right)^{r_{i}}
$$

Figure 6 compares the number of expected transmissions from a repair server to receivers in its repair domain using an APES approach to non-APES approaches as (a) the block size, $k$, varies, (b) the domain size varies, and (c) the high loss rate varies along the $x$-axis. As a default, the block size, $k$, equals ten, the domain size of each domain, $r_{i}$, is ten, and there are ten domains of type one with loss rates from repair server to receiver of $p_{1}=.01$, and a single domain of type two with loss rates of $p_{2}=.1$. In

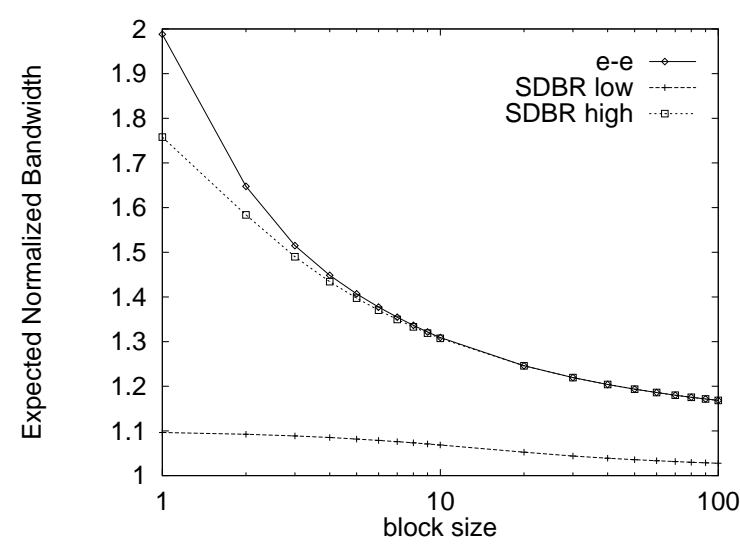

(a) Varying block size

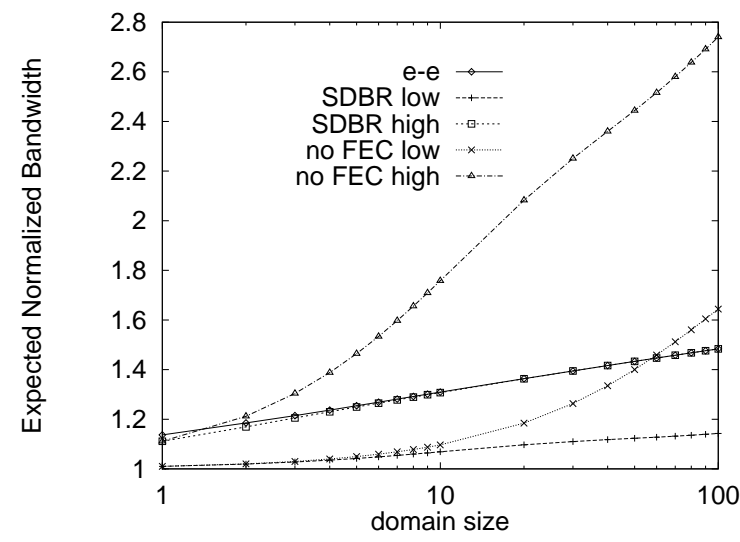

(b) Varying domain size

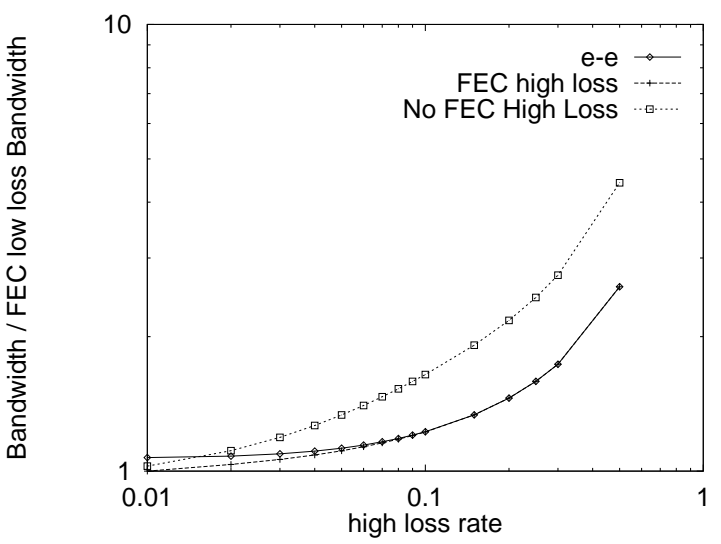

(c) Varying high loss rate

Fig. 6. Expected number of packets transmitted to receivers in interheterogeneous, intra-homogeneous networks varying (a) block size, (b) repair domain size, and (c) high loss rate.

Figures 6(a) and 6(b), the block size, $k$, and the domain size vary along the $x$-axis, respectively. Figure 6(a) plots curves, where the $y$-axis equals the normalized, expected cost of reliably transmitting a data packet. The curves labeled e-e, SDBR low, and SDBR high plot values of $E\left[N^{E}\right] / k, E\left[N_{1}^{S}\right] / k$, and $E\left[N_{2}^{S}\right] / k$, 
respectively. Note that values for $E\left[F_{1}\right]$ and $E\left[F_{2}\right]$ are given by respective plots SDBR low and SDBR high when the block size, $k$, equals one. Figure 6(b) includes additional plots for $E\left[F_{1}\right]$ and $E\left[F_{2}\right]$, respectively labeled no FEC low and no FEC high. In Figure 6(c), the loss rate in the type two, high loss, domain is varied along the $x$-axis, while the $y$-axis equals the normalized, expected cost of reliably transmitting a data packet in multiples of this cost in a low loss (type one) domain. Plots labeled e-e, FEC high loss, and No FEC high loss respectively plot values for $E\left[N^{E}\right] / E\left[N_{1}^{S}\right], E\left[N_{2}^{S}\right] / E\left[N_{1}^{S}\right]$, and $k E\left[F_{2}\right] / E\left[N_{1}^{S}\right]$.

From Figures 6(a) and 6(b), we see that the bandwidth used by the e-e protocol throughout the network is almost identical to what is required in the high loss domain, which means low loss domains incur significant additional bandwidth. In Figure 6(c), we see that in an intra-homogeneous, intra-heterogeneous network, there is a small savings in bandwidth due to the use of repair services. As we increase the inter-heterogeneity, adding FEC reduces bandwidth further in protocols that make use of repair services, particularly in high loss domains. This behavior is observed in Figure 6(a), as well as in Figure 6(b) by comparing SDBR and the RS protocol for either the high or low loss domain. We also observed little variation in bandwidth usage as the ratio of the number of low loss domains to the number of high loss domains, $n_{1} / n_{2}$, increases. The plot has been omitted due to lack of variation along $x$-axis.

We conclude from these observations that the bandwidth used throughout the network in an end-to-end FEC approach is dominated by the bandwidth required by domains with high loss, whereas in a network with repair servers, this bandwidth consumption can be limited to the domains where it is required. An additional savings in bandwidth comes as a result of using FEC, particularly in high loss or large domains. We expect the improvement due to FEC to be less if the loss between repair server and receivers has a high level of spatial correlation. However, our analysis in Section III showed that using FEC still improves performance by reducing buffering requirements.

\section{FUTURE WORK AND CONCLUSION}

We have performed our examination of APES protocols using a simplified network model. One direction for future work is to implement the protocols and compare their performance in a real networking environment. Since we now understand how certain basic characteristics affect performance, another direction is to use a model which more accurately captures a real networking environment. In particular, we have not directly considered loss between the source and repair servers, temporally correlated (bursty) loss, or spatially correlated loss. We point out that loss between the source and repair servers does not affect the bandwidth requirements between repair servers and receivers. Nor does it affect the buffer requirements of repair servers. Results in [3], [8] indicate that the levels of burstiness (i.e., temporally correlated loss) observed in today's Internet will cause a slight, often unnoticeable decrease in performance of FEC.

We now turn our attention to spatially correlated loss. Spatial correlation was examined in [9] for SDBR in intra-heterogeneous, inter-homogeneous environments. The techniques presented there can easily be applied to examine the effects of spatial correlation on inter-heterogeneous environments, and on BRSR and GRSR. We also point out that a domain size of one is equiv- alent to assuming that all losses within a domain are $100 \%$ correlated. Thus, the bandwidth utilized in a within a domain size of $r$ with partially correlated loss lies somewhere between the results plotted for a domain size of $r$ and those plotted for a domain size of one. While correlated loss will reduce the effectiveness of FEC in reducing bandwidth requirements, we can expect an increase in its effectiveness at reducing buffering requirements.

There is still considerable interest in reducing the bandwidth consumption of reliable multicast protocols. Two approaches that successfully achieve such reduction are repair services approaches and hybrid FEC/ARQ approaches. We have examined and compared performance of protocols that combine these approaches, which we call APES protocols. We described several new APES protocols that maintain this high bandwidth efficiency while reducing buffer and FEC processing requirements. We thoroughly explored how the bandwidth, buffer, and FEC processing requirements vary among the various versions of the protocols. We also describe the kinds of networking environments in which such protocols improve the bandwidth efficiency of reliable multicast beyond what either approach is able to accomplish separately.

\section{ACKNOWLEDGMENT}

We would like to thank Steve Zabele of TASC, who suggested that we consider on-the-fly encoding techniques, and the anonymous reviewers for their helpful comments.

\section{REFERENCES}

[1] J. Blomer, M. Kalfane, R. Karp, M. Karpinski, M. Luby, and D. Zuckerman, An XOR-Based Erasure-Resilient Coding Scheme, International Computer Sciences Institute Technical Report ICSI TR-95-048, August 1995.

[2] J. Byers, M. Luby, M. Mitzenmacher, and A. Rege, A Digital Fountain Approach to Reliable Distribution of Bulk Data, Proceedings of ACM SIGCOMM'98, Vancouver, CA, September 1998

[3] M. Handley, An Examination of MBone Performance, Technical Report, UCL and ISI, January 1998.

[4] S. Kasera, J. Kurose, D. Towsley, A Comparison of Server-Based and Receiver-Based Local Recovery Approaches for Scalable Reliable Multicast, UMass CMPSCI Technical Report 97-69. A shorter version of this report appeared in IEEE INFOCOM98.

[5] R. Kermode, Scoped Hybrid Automatic Repeat Request with Forward Error Correction (SHARQFEC), Proceedings of ACM SIGCOMM'98, Vancouver, CA, September 1998.

[6] L.H. Lehman, S.J. Garland, and D.L. Tennenhouse, Active Reliable Multicast, Proceedings of IEEE INFOCOM98, San Francisco, CA, March, 1998.

[7] J. C. Lin and Sanjoy Paul. Reliable Multicast Transport Protocol (RMTP), IEEE JSAC, 407, 3, 1414-1424,April 1997.

[8] J. Nonnenmacher, E. Biersack, and D. Towsley, Parity-Based Loss Recovery for Reliable Multicast Transmission, Proceedings of ACM SIGCOMM '97, Sept. 1997 Cannes, France, pp. 289-300.

[9] J. Nonnenmacher, M. Lacher, M. Jung, E. W. Biersack and Georg Carle, How bad is reliable multicast without local recovery?, Proceedings of IEEE INFOCOM'98, San Francisco, CA, USA, March 1998.

[10] Luigi Rizzo, Effective Erasure Codes for Reliable Computer Communication Protocols, Computer Communication Review, April 1997.

[11] Dan Rubenstein, Sneha Kasera, Don Towsley, and Jim Kurose, Improving Reliable Multicast Using Active Parity Encoding Services (APES), UMass CMPSCI Technical Report 98-79.

[12] D. Towsley, J. Kurose, S. Pingali, A Comparison of Sender-Initiated and Receiver-Initiated Reliable Multicast Protocols, IEEE Journal on Selected Areas in Communications, April 1997.

[13] B. Whetten et al, The RMTP-II Protocol, Internet Draft, April, 1998.

[14] M. Yajnik, J. Kurose, D. Towsley, Packet Loss Correlation in the MBone Multicast Network, Proceedings of IEEE Global Internet Conference. London, November 1996. 